\title{
Allicin inhibits cell growth and induces apoptosis in U87MG human glioblastoma cells through an ERK-dependent pathway
}

\author{
JAE HUN CHA ${ }^{1}$, YOON JI CHOI ${ }^{2}$, SEUNG HEON CHA ${ }^{1}$, CHANG HWA CHOI $^{1}$ and WON HO CHO ${ }^{1,2}$ \\ ${ }^{1}$ Department of Neurosurgery and ${ }^{2}$ Medical Research Institute, Pusan National University Hospital, \\ Pusan National University School of Medicine, Busan 602-739, Republic of Korea
}

Received December 16, 2011; Accepted March 6, 2012

DOI: $10.3892 / o r .2012 .1772$

\begin{abstract}
Allicin, the main flavor compound in garlic, has anti-carcinogenic activities in a range of cancer cells, however, the underlying molecular mechanisms are not completely understood. This study examined the effect of allicin on the cell viability of U87MG human glioma cells along with its molecular mechanisms of induction of cell death. Apoptosis was determined by TUNEL and Hoechst 33258 staining as well as by western blot analysis. Allicin inhibited the cell viability of U87MG human glioma cells in a dose- and time-dependent manner. Allicin-induced inhibition of cell viability was due to apoptosis of cells. The mechanisms of apoptosis were found to involve the mitochondrial pathway of Bcl-2/Bax, the MAPK/ ERK signaling pathway and antioxidant enzyme systems. These results suggest that allicin can serve as a novel chemotherapeutic candidate for the treatment of glioblastoma multiforme.
\end{abstract}

\section{Introduction}

Glioblastoma multiforme (GBM) is one of the most malignant and aggressive of human cancers (1-3). Although there have been marked advancements in microsurgical techniques, radiotherapy and chemotherapy, the prognosis of GBM remains dismal with a median survival of 1 year. Moreover, no new treatment modalities, such as gene therapy and novel chemotherapeutic agents, have achieved favorable results (4-6).

Allicin (2-propene-1-sulfinothioic acid S-2-propenyl ester) is the main flavor compound in freshly crushed garlic

Correspondence to: Dr W.H. Cho, Department of Neurosurgery, Pusan National University Hospital, Pusan National University School of Medicine, Busan 602-739, Republic of Korea

E-mail:mdcwh@naver.com

Abbreviations: GBM, glioblastoma multiforme; MTT, [(3-(4, 5-dimethylthiazol-2-yl)-2, 5-diphenyltetrazolium bromide]; MAPK, mitogen-activated protein kinase; ERK, extracellular signal-regulated kinase; JNK, c-Jun NH2-terminal kinase; NAC, N-acetylcysteine; ROS, reactive oxygen species

Key words: allicin, apoptosis, extracellular signal-regulated kinase, antioxidants, U87MG cells
(Allium sativum), and has been investigated for its anti-cancer, anti-viral and anti-inflammatory activities (7-9). Moreover, the anti-cancer effect of allicin has been demonstrated in a range of cancer cells including SiHa cells (human cervical cancer cell line) (10), HepG2 (hepatocellular carcinoma cell line) (11-14), SGC-7901 (gastric carcinoma cell line) (15) and MCF-7 cells (human breast cancer cell line) (16). According to these experiments, allicin is expected to be a novel anti-cancer or cancer-preventive agent but its effects on GBM are unclear.

This study examined the effect of allicin on the cell viability of U87MG human glioma cells, as well as the molecular mechanisms including the signaling pathway related to apoptosis under various allicin-treated conditions.

\section{Materials and methods}

Reagents. Allicin was obtained from NPC Bio Tech (Korea). Hoechst 33258, dimethylsulfoxide (DMSO), DAPI staining, and 3-(4,5-dimethylthiazol-2-yl)-2, 5-diphenyltetrazolium bromide (MTT) was purchased from Sigma-Aldrich (USA). RPMI-1640 medium, phosphate-buffered saline (PBS) and fetal bovine serum (FBS) were from Invitrogen (Canada). U0126, PD98053, SP600125, SB203580 and Z-VAD-fmk were obtained from Calbiochem (DEN). Enhanced chemiluminescence kit (ECL) was obtained from Amersham Biosciences (Germany). Bcl-2, Bax and Pro-caspase-3 were obtained from Epitomics (USA). p-ERK, p-p38 and p-JNK were purchased from Cell Signaling Technology Inc. (USA).

Cell line and culture conditions. U87MG cells (human glioblastoma cell line) were purchased from Korean Cell Line Bank (Korea), and cultured in RPMI-1640 medium containing $10 \%$ heat-inactivated FBS. The cells were seeded in culture dishes at $37^{\circ} \mathrm{C}$ in humidified $5 \% \mathrm{CO}_{2}$ incubator. These cells were cultured for 3-5 days until confluence and sub-cultured using $0.05 \%$ trypsin solution and seeded in 6- or 96-well tissue culture plates. Serum was starved from culture media before various reagent additions for 1-2 days.

Measurement of cell growth by MTT. Cell viability was assessed using an MTT [3-(4,5-dimethylthiazol-2-yl)-2, 5-diphenyltetrazolium bromide] assay. U87MG cells were plated in 96-well plates at $5 \times 10^{2}$ cells per well and indicated with various concentrations of allicin and time periods. In brief, cells were washed 

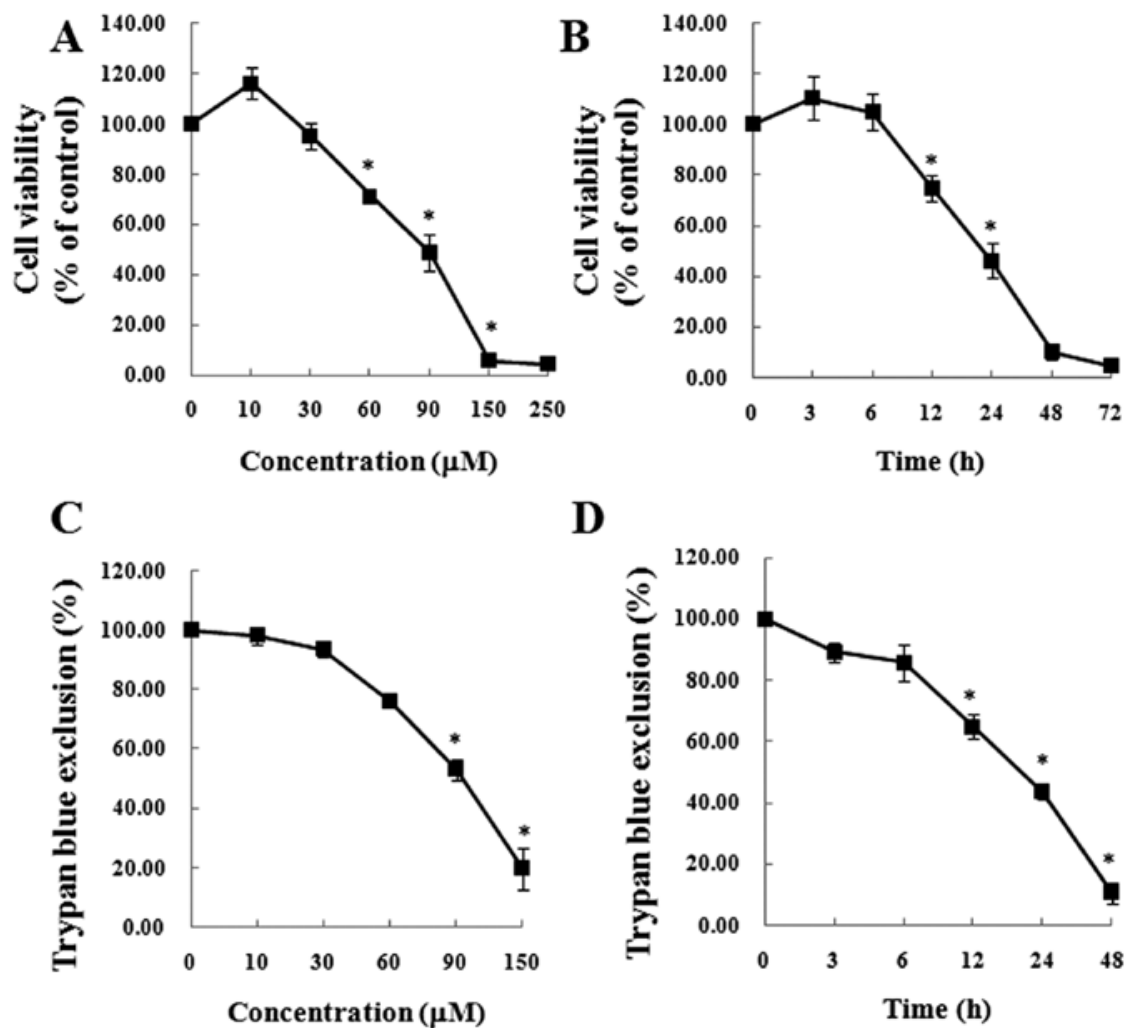

D

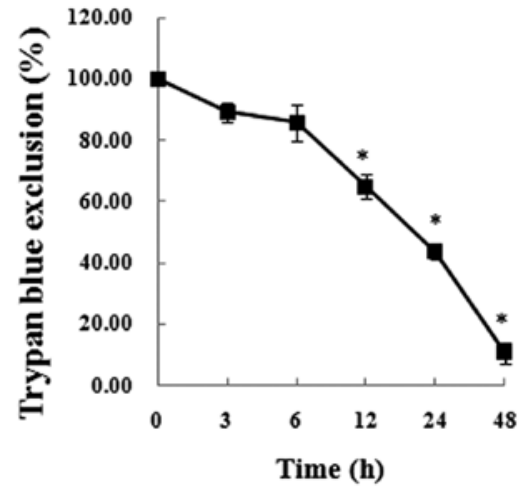

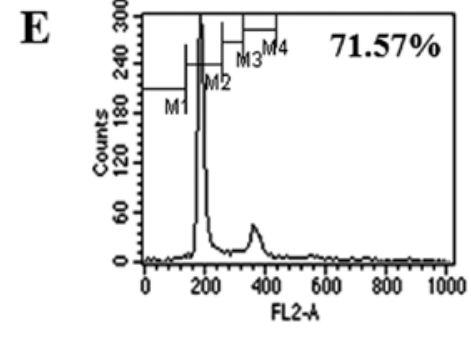

Cont.

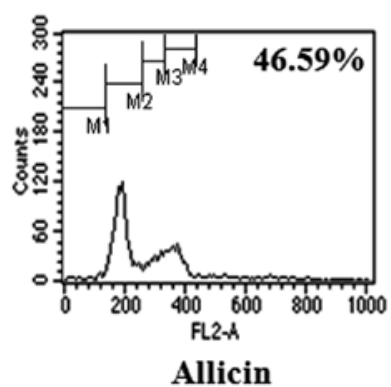

Figure 1. Inhibitory effect of allicin on U87MG cells. The viability of U87MG cells treated with various concentrations of allicin for $24 \mathrm{~h}$ measured using a MTT assay (A), trypan blue exclusion (C) and the viability of U87MG cells treated with $90 \mu \mathrm{M}$ allicin for various times (B) and (D). The cells were treated with $90 \mu \mathrm{M}$ allicin for $24 \mathrm{~h}$ analyzed by flow cytometry. The percentage means a G0/G1 peak indicated by M2 (E). The data are reported as the mean \pm SEM of four independent experiments. ${ }^{*} \mathrm{P}<0.05$ compared to the control without allicin.

after treatment with various conditions of allicin and $0.5 \mathrm{mg}$ $\mathrm{MTT} / \mathrm{ml}$ in RPMI-1640 medium was added. After $2 \mathrm{~h}$ at $37^{\circ} \mathrm{C}$, cells were dissolved in DMSO. The absorbance at $570 \mathrm{~nm}$ was measured with 680 micro-plates ELISA reader (Germany).

Flow cytometry. Cells were seeded in 6-well plates at $5 \times 10^{4}$ cells per well, treated with the reagents for $24 \mathrm{~h}$ at $37^{\circ} \mathrm{C}$ and then harvested using $0.05 \%$ trypsin solution and centrifuged at $10000 \mathrm{x} \mathrm{g}$ for $15 \mathrm{~min}$. The pellets were washed in PBS buffer two times and added to fixing solution, overnight at $4^{\circ} \mathrm{C}$. After fixation, cells were stained with $50 \mu \mathrm{g}$ propidium iodide $/ \mathrm{ml}$ contained $100 \mu \mathrm{g}$ RNase $/ \mathrm{ml}$ for $20 \mathrm{~min}$ at $37^{\circ} \mathrm{C}$ and analyzed using FACSort Becton-Dickinson flow cytometer (USA).

\section{Staining of the apoptotic cells}

TUNEL assay. The cells were plated in cover slides at $5 \times 10^{2}$ cells, and then treated with allicin. The cells were washed in PBS buffer and freshly prepared $4 \%$ paraformaldehyde was added for $60 \mathrm{~min}$ in $37^{\circ} \mathrm{C}$ in humidified $5 \% \mathrm{CO}_{2}$ incubator. Cells were washed and permeabilized in solution of $0.1 \%$ Triton X-100 in $0.1 \%$ sodium citrate, for $2 \mathrm{~min}$ on ice. And then cells were subjected to the TUNEL reaction at $37^{\circ} \mathrm{C}$ in a humidified atmosphere in the dark for $60 \mathrm{~min}$. The fluorescent signal was detected by fluorescence microscope (Germany). DNA fragmentation presence was evaluated by the terminal deoxynucleotidyl transferase (TdT)-mediated deoxyuridine triphosphate (dUTP) nick-end labeling (TUNEL) assay using the In Situ Cell Death Detection kit (fluorescein) purchased from Roche Applied Science (USA).

Cytochemical staining (Hoechst 33258 staining). The cells were seeded in cover slides at $5 \times 10^{2}$ cells, and then treated with allicin. The cells were fixed with freshly prepared $4 \%$ paraformaldehyde for $20 \mathrm{~min}$ at $4^{\circ} \mathrm{C}$ and then permeabilized in solution of $0.3 \%$ 
Triton X-100 in PBS for 5 min. Cells were stained with $20 \mu \mathrm{M}$ Hoechst 33258 at room temperature in the dark for $30 \mathrm{~min}$. The fluorescent signal was detected by a fluorescence microscope.

Measurement of cell death by a trypan blue dye exclusion assay. Allicin treated cells were harvested using $0.05 \%$ trypsin solution, suspension with $0.4 \%$ trypan blue solution. The cells were counted using a hemocytometer under light microscopy. The cells which excluded the dye were considered viable.

Immunocytochemistry. The cells were seeded in cover glass slides at $5 \times 10^{2}$ cells $/ \mathrm{cm}^{2}$ and treated with allicin. Cells were fixed in freshly prepared $4 \%$ paraformaldehyde for $5 \mathrm{~min}$ on ice and then washed, blocked in $1 \%$ BSA blocking reagent for $30 \mathrm{~min}$ at room temperature. Next, cells were stained with primary antibodies Bax (1:500) and Bcl-2 (1:300) overnight at $4^{\circ} \mathrm{C}$ and then washed with PBS buffer and the secondary antibody was added for $2 \mathrm{~h}$. Cell nuclei were stained using DAPI staining.

Western blot analysis. The cells were seeded in 6-well plates at $5 \times 10^{4}$ cells $/ \mathrm{cm}^{2}$. Using lysis buffer $(10 \mathrm{mM} \mathrm{NaCl}, 20 \mathrm{mM}$ Tris-HCl, 1 mM EGTA, $1 \mathrm{mM}$ EDTA, $0.1 \mathrm{mM}$ phenylmethylsulfonyl fluoride, $1 \%$ Triton X-100, pH 7.4), cells were lysed on ice. Lysates were centrifuged at $10000 \mathrm{x} \mathrm{g}$ for $20 \mathrm{~min}$ at $4^{\circ} \mathrm{C}$ and the supernatants containing equal amounts of protein were loaded on $15 \%$ SDS-PAGE (sodium dodecyl sulfate polyacrylamide gel electrophoresis) gels, transferred, and immunoblotted with various antibodies. The membrane signals were visualized using an ECL kit.

Statistical analysis. All experiments were performed at least three times. Statistical significance was analyzed using a Student's t-test (two-tailed). A p $<0.05$ was considered statistically significant.

\section{Results}

Inhibitory effect of allicin on the cell viability. The inhibitory effects of allicin on the cell viability of U87MG human glioma cells were examined using an MTT [(3-(4,5-dimethylthiazol2-yl)-2, 5-diphenyltetrazolium bromide] assay and trypan blue exclusion assay. The cells were treated with allicin under a range of concentrations and time periods. Allicin induced a decrease in cell viability in a dose- (Fig. 1A and C) and time-dependent manner (Fig. 1B and D). Flow cytometry was performed to re-affirm the inhibitory effect of allicin on U87MG cells. The allicin treatment induced a decrease in the G0/G1 peak from 71.57 to $46.59 \%$ (Fig. 1E). These results provide evidence of inhibitory effects of allicin on the viability of U87MG cells.

Effect of allicin on the cell morphology. The cells were treated with $90 \mu \mathrm{M}$ allicin, and the changes in morphology were visualized by light microscopy. The allicin-treated cells exhibited cell rounding and shrinkage (Fig. 2).

Allicin induces apoptosis on U87MG cells. The effects of allicin on the cell viability and proliferation of U87MG human glioma cells were examined using a range of methods. The human glioblastoma cells exposed to allicin were examined by western blot analysis and a TUNEL assay to identify the type of cell death. As shown in Fig. 3A, the number of positively stained (green) cells was increased significantly under allicin-treatment conditions compared to the untreated condition. The cells revealed apoptotic changes in the nucleus according to Hoechst 33258 staining (Fig. 3B).

The cells treated with allicin were stained with a pro-apoptotic member, Bax, and an anti-apoptotic member, Bcl-2, and examined by immunocytochemistry (Fig. 3C). The expression of Bax was positive (green), whereas the level of Bcl-2 expression was very low. The effect of allicin in the cells was examined by western blot analysis to confirm the data in Fig. 3. The expression of the Bax protein was increased but that of $\mathrm{Bcl}-2$ was decreased in a dose- and time-dependent manner (Fig. 4). This suggests that allicin induces apoptosis in U87MG cells.

Allicin-induced apoptosis is regulated by MAPK/ ERK-dependent pathway. Allicin was reported to induce apoptosis in human glioblastoma cells that was regulated by a range of signaling pathways including mitogen-activated protein kinase (MAPK) pathway, caspase-mediated pathway and mitochondrial pathway (11-13).

The involvement of the MAPKs family in allicin-induced apoptosis was examined. Activation of the MAPKs subfamily was examined by western blot analysis using specific antibodies, such as phosphor-EKR (p-ERK), -p38 (p-p38), -JNK (p-JNK). p-ERK and p-p38 expression were detected but p-JNK was not activated (Fig. 5A). The involvement of the MAPK pathway was examined by pretreating the cells with each MAPK inhibitor and assessing the cell viability using an MTT assay and trypan blue exclusion analysis (Fig. 5B and C). Allicin-induced cell death was prevented by a pre-treatment with U0126 (inhibitor of ERK). Flow cytometry analysis was performed after the U0126 pre-treatment to confirm the results (Fig. 5D). Cell proliferation was sustained by the U0126 pre-treatment compared with the control. These results provide evidence of allicin-induced apoptosis regulated by the ERK-dependent pathway.

Allicin-induced apoptosis is regulated by antioxidant enzymes. Based on the preventive effect of antioxidant enzymes to malignancies, antioxidants have long been researched as candidates for cancer therapy (17-19). The U87MG cells were pre-treated with antioxidants, NAC (N-acetyl-L-cysteine) and catalase, to determine the effect of antioxidant enzymes. In the pre-treated group, the cell viability was not reduced compared to the control group (Fig. 6A and B). Flow cytometry demonstrated an increase in the signal compared to the allicin treatment alone (Fig. 6C). This suggests that allicin-induced apoptosis is regulated by antioxidant enzymes.

Allicin-induced apoptosis is not regulated by the caspase cascade. The cells were exposed to the general caspase inhibitor, Z-VAD-fmk, to identify the involvement of the caspase cascade in allicin-induced apoptosis. Allicin-induced apoptosis was reported to be regulated through the caspase-dependent or -independent pathway $(20,21)$. Fig. 7 show that Z-VAD-fmk did not affect the cell viability. Similar results were obtained by western blot analysis, and comparable levels of Bcl-2 and Bax expression were observed (data not shown). In summary, the caspase cascade is not related to allicin-induced apoptosis. 


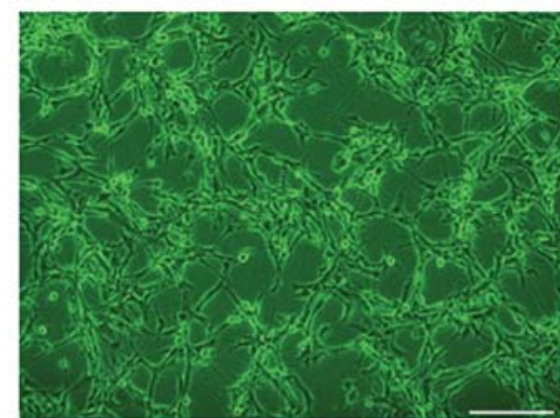

Cont.

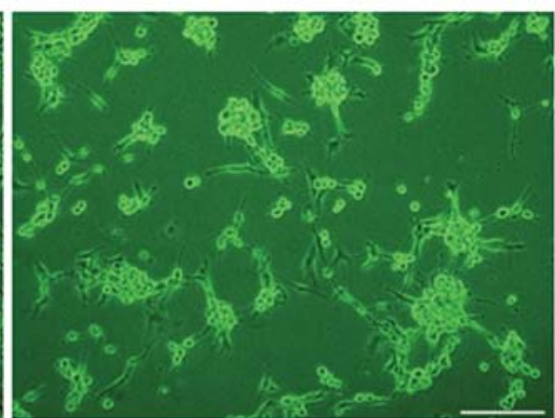

Allicin

Figure 2. Morphological changes by allicin in U87MG cells. The cells showed morphological changes after a 24-h treatment with $90 \mu \mathrm{M}$ allicin. Scale bar, $400 \mu \mathrm{m}$.

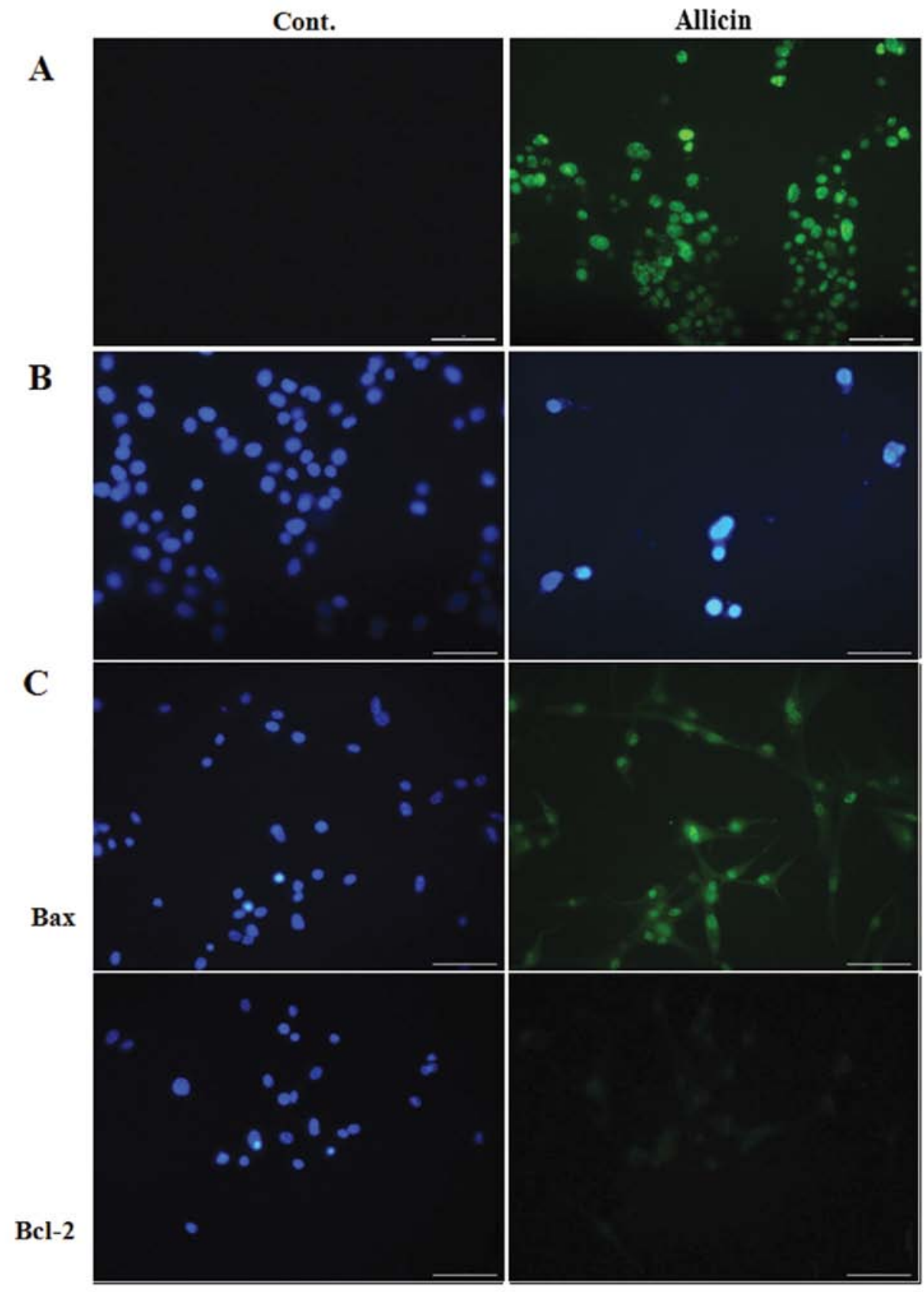

Figure 3. Allicin induces apoptosis in U87MG cells. The cells were treated with $90 \mu \mathrm{M}$ allicin for $24 \mathrm{~h}$ and examined using a TUNEL assay (A), Hoechst 33258 stain (B), and immunocytochemistry. Left panel, DAPI staining (C). Scale bar, $400 \mu \mathrm{m}$. 
A

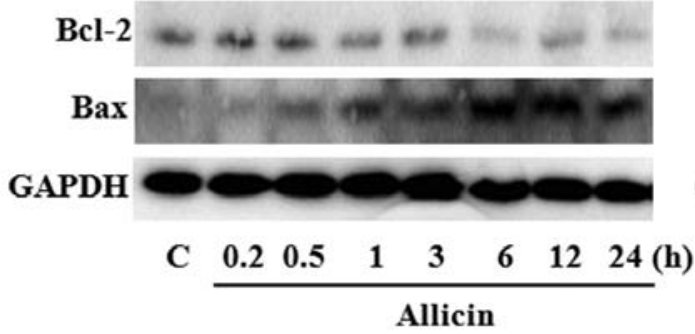

B

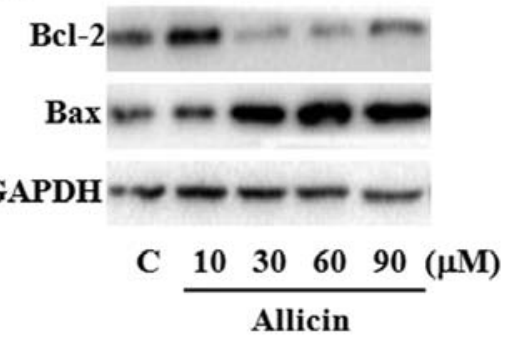

Figure 4. Allicin induces apoptosis in U87MG cells. The cells were treated with various concentrations of allicin for $24 \mathrm{~h}(\mathrm{~A})$ and times with $90 \mu \mathrm{M}$ allicin (B). The Bax and Bcl-2 expression were detected by western blot analysis. The experiments were performed in triplicate.

A

p-p38

p-ERK

GAPDH

C \begin{tabular}{llllllll}
0.2 & 0.5 & 1 & 3 & 6 & 12 & 24 & (h) \\
\hline Allicin & &
\end{tabular}

B

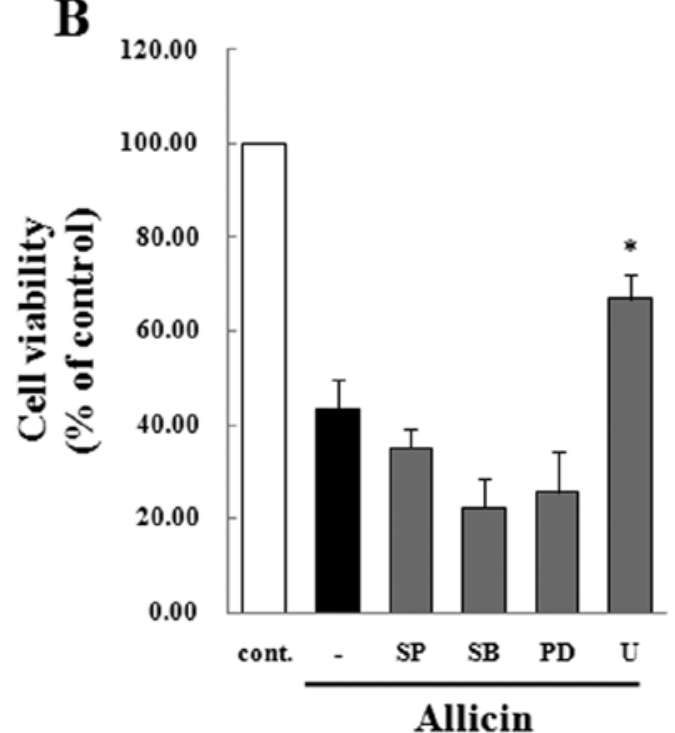

C 120.00
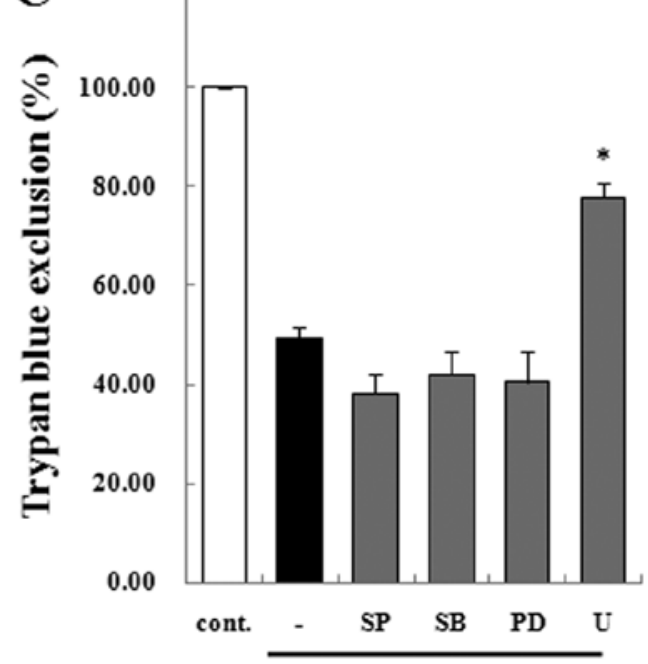

Allicin

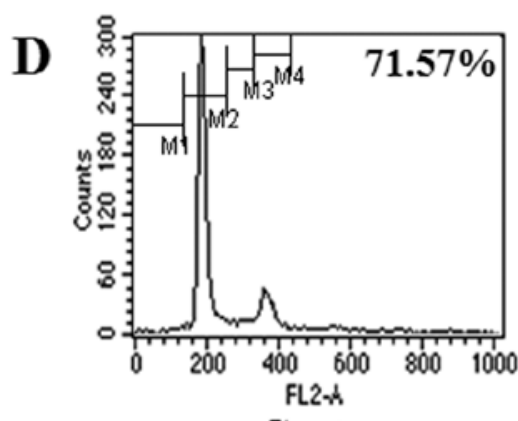

Cont.

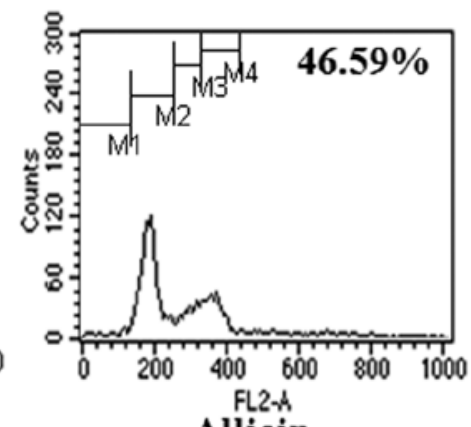

Allicin

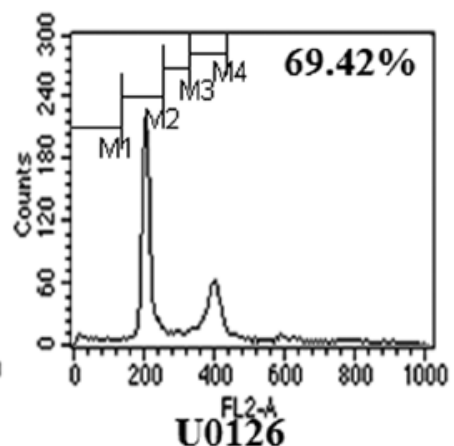

Figure 5. MAPK/ERK pathways involved in allicin-induced apoptosis. The activation of p-ERKnd p-p38 in the cells treated with $90 \mu \mathrm{M}$ allicin was detected by western blot analysis (A). The cells were pre-treated with U0126 $20 \mu \mathrm{M}$ (U), SB203580 $20 \mu \mathrm{M}$ (SB), SP $60012525 \mu \mathrm{M}$ (SP), and PD $9805920 \mu \mathrm{M}$ (PD) for $60 \mathrm{~min}$ then treated with $90 \mu \mathrm{M}$ allicin for $24 \mathrm{~h}$. The cell viability was measured using an MTT assay (B) and trypan blue exclusion assay (C). The cells were pre-treated with $20 \mu \mathrm{M}$ of U0126 for 60 min then treated with $90 \mu \mathrm{M}$ allicin and subjected to flow cytometry. The percentage of G0/G1 peak is indicated by M2 (D). The data are reported as the mean \pm SEM of four independent experiments. * $\mathrm{P}<0.05$ compared to allicin alone. 
A

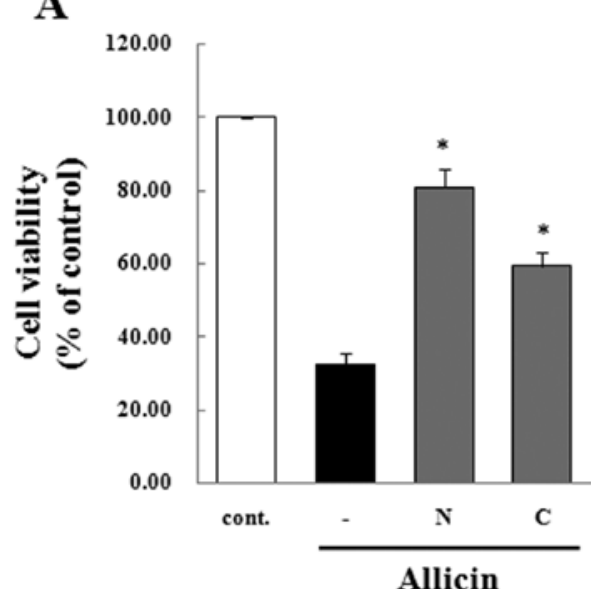

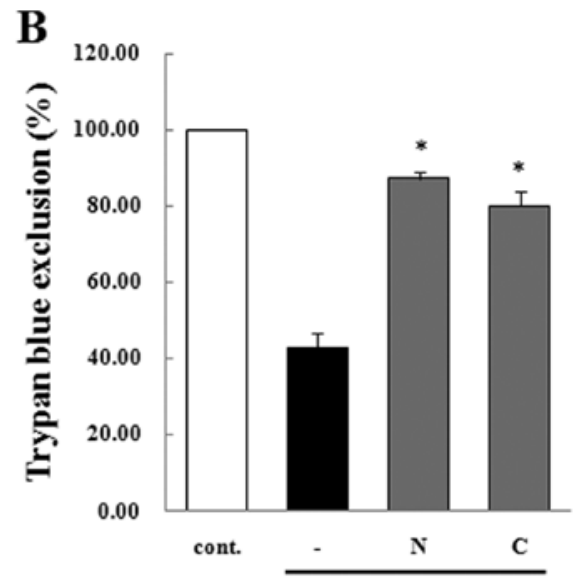

Allicin
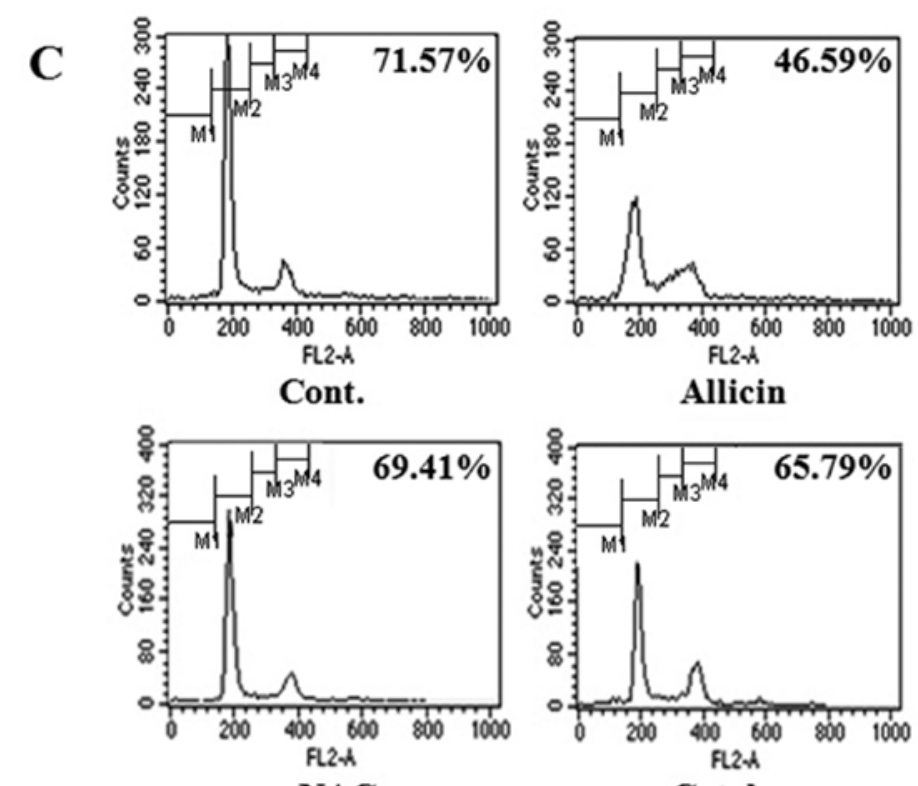

Figure 6. Antioxidant enzyme system involved in the allicin-induced apoptosis. The cells were pre-treated with NAC $10 \mu \mathrm{M}(\mathrm{N})$ and catalase $800 \mathrm{U} / \mathrm{ml}(\mathrm{C})$ for $90 \mathrm{~min}$ and then treated with allicin $90 \mu \mathrm{M}$ for $24 \mathrm{~h}$. The cell viability was measured using an MTT assay (A), trypan blue exclusion assay (B) and flow cytometry. The percentage means the G0/G1 peak indicated by M2 (C). The data are reported as the mean \pm SEM of four independent experiments. "P<0.05 compared to allicin alone.
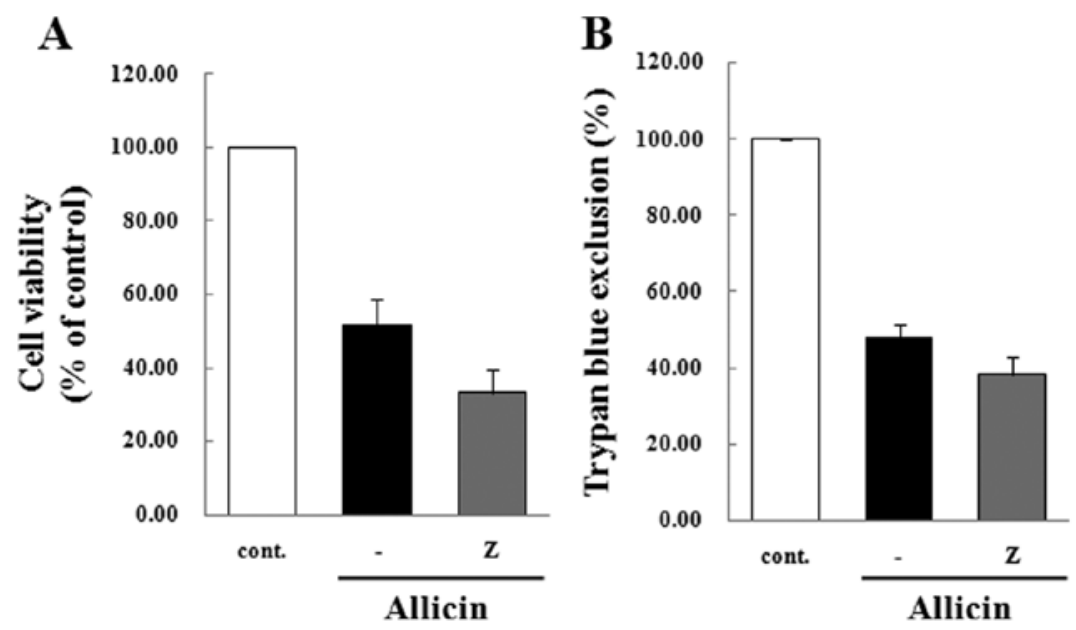

Figure 7. Caspase cascade is not involved in allicin-induced apoptosis. The cells were pre-treated with $20 \mu \mathrm{M} \mathrm{Z-VAD-fmk} \mathrm{(Z)} \mathrm{for} 60 \mathrm{~min}$ treated with $90 \mu \mathrm{M}$ allicin for $24 \mathrm{~h}$ and the cell viability was measured using an MTT assay (A), trypan blue exclusion assay (B). The data are reported as the mean \pm SEM of four independent experiments. 


\section{Discussion}

Although many novel trials for the treatment of glioblastoma multiforme (GBM) have been reported, GBM still remains one of the most malignant neoplasms in humans. Its prognosis is extremely poor with a mean survival of less than 1 year. Recent studies have focused on the development of new treatment modalities for GBM, such as gene therapy, biological therapy, hormone therapy, chemotherapy and natural substances from a range of foods (22-24). Among them, attempts have been made to find new materials from natural food substances with anti-cancer effect for chemotherapy. Some studies reported that allicin inhibits the growth of various cancer cells and might play a major role as an anti-cancer agent. Moreover, allicin reduces the risk of cancer development, such as melanoma and leukemia $(16,25,26)$. Therefore, this study evaluated allicin as a potential a new chemotherapeutic agent of GBM.

The aim of this study was to determine the anti-cancer effect of allicin on GBM with U87MG cells. The molecular mechanisms involved in the allicin-induced anti-cancer effect in U87MG cells were examined. The cell viability of U87 MG cells was reduced by allicin in a dose- and time-dependent manner (Fig. 1). In addition, the allicin-induced inhibitory effect on cell viability of U87MG cells was verified by the morphological changes to the cells, such as shrinkage (Fig. 2).

The mechanism of allicin-induced cell death of U87MG cells, either apoptosis or necrosis, was examined. Apoptosis is a well-known process of programmed cell death by many stimuli (27-29). This process occurs in multi-cellular organisms and is regulated by many biological cellular processes, such as tissue growth, elimination of redundant cells, and immune responses. The occurrence of apoptosis in U87 MG cells was verified by TUNEL and Hoechst staining. TUNEL-positive stained cells and Hoechst-positive stained cells were increased by the allicin treatment (Fig. 3A and B). TUNEL assays, Hoechst 33258 staining and flow cytometry are common methods for detecting apoptosis (30-33).

Allicin-induced apoptosis was confirmed by measuring the levels of Bax and Bcl-2 expression. The analysis revealed an increase in Bax expression and a decrease in Bcl-2 expression (Fig. 4A and B). The Bcl-2 family might be involved in apoptosis through an interaction between the pro- and anti-apoptotic proteins (34-37). The down- and up-regulated expression of Bcl-2 and Bax, respectively, suggest that allicin-induced apoptosis is related to the mitochondrial pathway.

The activation or inhibition of various pathways was investigated to determine the precise molecular signaling mechanisms involved in allicin-induced apoptosis. The representative molecular mechanisms involved in apoptosis include the mitochondrial pathway, activation of mitogen-activated protein kinases (MAPKs), and caspase cascade and oxidant enzyme system. MAPKs, the principal intracellular signal transduction system, play key roles in cell survival, proliferation, differentiation and cell death $(11-13,38)$. MAPKs are comprised of the three parallel kinase modules, including p38-MAPK, ERK and JNK, especially the MAPK/ERK signaling pathway involved in cell survival and proliferation. Some active components from garlic have anti-cancer effects through the ERK1/2 and p38-MAPK signaling pathways $(20,39,40)$. In the present study, the level of ERK phosphorylation was increased, and allicin-induced apop- tosis was prevented by a pre-treatment with U0126, an ERK inhibitor (Fig. 5). This suggests that allicin-induced apoptosis is regulated by the ERK signaling pathway.

Reactive oxygen species (ROS) are associated with apoptosis (41-43). ROS interact with a range of cellular components, causing potential damage to membranes and other cellular structures. Antioxidant enzymes protect the cells from oxidative damage. This study demonstrated that allicin-induced apoptosis was down-regulated by the antioxidant enzyme system (Fig. 6). Therefore, ROS are related to allicin-induced apoptosis in the U87MG cells.

Caspase, an interleukin-1 $\beta$-converting enzyme family of proteases, are homologous to the caenorhabditis elegans cell death gene, CED-3. The caspase cascade is also one of the main molecular mechanisms during the execution phase in various forms of the apoptotic cascade (44). On the other hand, inhibition of the caspase activity did not prevent the apoptotic reaction in some cancer cells. The data also demonstrated that the caspase cascade did not mediate allicin-induced apoptosis (Fig. 7).

In conclusion, this study demonstrated that allicin inhibits the cell viability of U87MG human glioma cells and induces cell death through apoptosis. Allicin-induced apoptosis is mediated through the Bcl-2/Bax mitochondrial pathway, MAPK/ERK signaling pathway and antioxidant enzyme systems. Therefore, allicin can be a novel therapeutic agent for GBM. Nevertheless, more study is needed to establish the precise molecular mechanisms of allicin-induced apoptosis and apply allicin for the anti-tumoral effect in vivo.

\section{References}

1. Kelly KA, Kirkwood JM and Kapp DS: Glioblastoma multiforme: pathology, natural history and treatment. Cancer Treat Rev 11: 1-26, 1984.

2. Kala M, Srámek V, Houdek M, Vaverka M and Zmrzlík P: [Treatment of glioblastoma multiforme]. Cas Lek Cesk 132: 653-656, 1993.

3. Holland EC: Glioblastoma multiforme: the terminator. Proc Natl Acad Sci USA 97: 6242-6244, 2000.

4. Jendrossek V, Belka C and Bamberg M: Novel chemotherapeutic agents for the treatment of glioblastoma multiforme. Expert Opin Investig Drugs 12: 1899-1924, 2003.

5. Kanu OO, Mehta A, Di C, et al: Glioblastoma multiforme: a review of therapeutic targets. Expert Opin Ther Targets 13: 701-718, 2009.

6. Cheema FA, Badr A and Iqbal J: Glioblastoma multiforme presenting as treatment-resistant depression. J Neuropsychiatry Clin Neurosci 22: 123.E126, 2010.

7. Agarwal KC: Therapeutic actions of garlic constituents. Med Res Rev 16: 111-124, 1996.

8. Butt MS, Sultan MT and Iqbal J: Garlic: nature's protection against physiological threats. Crit Rev Food Sci Nutr 49: 538-551, 2009.

9. Lissiman E, Bhasale AL and Cohen M: Garlic for the common cold. Cochrane Database Syst Rev: CD006206, 2009.

10. Oommen S, Anto RJ, Srinivas G and Karunagaran D: Allicin (from garlic) induces caspase-mediated apoptosis in cancer cells. Eur J Pharmacol 485: 97-103, 2004.

11. Seger R and Krebs EG: The MAPK signaling cascade. FASEB J 9: 726-735, 1995

12. Lee S, Lee HS, Baek M, et al: MAPK signaling is involved in camptothecin-induced cell death. Mol Cells 14: 348-354, 2002.

13. Roux PP and Blenis J: ERK and p38 MAPK-activated protein kinases: a family of protein kinases with diverse biological functions. Microbiol Mol Biol Rev 68: 320-344, 2004.

14. Zhang ZM, Yang XY, Deng SH, Xu W and Gao HQ: Antitumor effects of polybutylcyanoacrylate nanoparticles of diallyl trisulfide on orthotopic transplantation tumor model of hepatocellular carcinoma in BALB/c nude mice. Chin Med J (Engl) 120: 1336-1342, 2007. 
15. Sun L and Wang X: Effects of allicin on both telomerase activity and apoptosis in gastric cancer SGC-7901 cells. World J Gastroenterol 9: 1930-1934, 2003.

16. Hirsch K, Danilenko M, Giat J, et al: Effect of purified allicin, the major ingredient of freshly crushed garlic, on cancer cell proliferation. Nutr Cancer 38: 245-254, 2000.

17. Prasad K, Laxdal VA, Yu M and Raney BL: Antioxidant activity of allicin, an active principle in garlic. Mol Cell Biochem 148 183-189, 1995.

18. Oberley TD and Oberley LW: Antioxidant enzyme levels in cancer. Histol Histopathol 12: 525-535, 1997.

19. Kong Q and Lillehei KO: Antioxidant inhibitors for cancer therapy. Med Hypotheses 51: 405-409, 1998.

20. Park SY, Cho SJ, Kwon HC, Lee KR, Rhee DK and Pyo S: Caspaseindependent cell death by allicin in human epithelial carcinoma cells: involvement of PKA. Cancer Lett 224: 123-132, 2005.

21. Miron T, Wilchek M, Sharp A, et al: Allicin inhibits cell growth and induces apoptosis through the mitochondrial pathway in HL60 and U937 cells. J Nutr Biochem 19: 524-535, 2008.

22. Shrieve DC, Alexander E, Black PM, et al: Treatment of patients with primary glioblastoma multiforme with standard postoperative radiotherapy and radiosurgical boost: prognostic factors and long-term outcome. J Neurosurg 90: 72-77, 1999.

23. Henson JW: Treatment of glioblastoma multiforme: a new standard. Arch Neurol 63: 337-341, 2006.

24. Chamberlain MC: Treatment options for glioblastoma. Neurosurg Focus 20: E19, 2006.

25. Patya M, Zahalka MA, Vanichkin A, et al: Allicin stimulates lymphocytes and elicits an antitumor effect: a possible role of p21 $1^{\text {ras. }}$. Int Immunol 16: 275-281, 2004.

26. Kyung KH: Antimicrobial properties of allium species. Curr Opin Biotechnol: Sep 6, 2011 (Epub ahead of print).

27. Kerr JF, Wyllie AH and Currie AR: Apoptosis: a basic biological phenomenon with wide-ranging implications in tissue kinetics. Br J Cancer 26: 239-257, 1972.

28. Hickman JA: Apoptosis induced by anticancer drugs. Cancer Metastasis Rev 11: 121-139, 1992.

29. Yue TL, Ohlstein EH and Ruffolo RR: Apoptosis: a potential target for discovering novel therapies for cardiovascular diseases. Curr Opin Chem Biol 3: 474-480, 1999.
30. Negoescu A, Guillermet C, Lorimier P, Brambilla E and LabatMoleur F: Importance of DNA fragmentation in apoptosis with regard to TUNEL specificity. Biomed Pharmacother 52: 252-258, 1998.

31. Nagata S: DNA degradation in development and programmed cell death. Annu Rev Immunol 23: 853-875, 2005.

32. Wieder R: TUNEL assay as a measure of chemotherapy-induced apoptosis. Methods Mol Med 111: 43-54, 2005.

33. Hewitson TD and Darby IA: In situ localization of apoptosis using TUNEL. Methods Mol Biol 611: 161-170, 2010.

34. Gross A, McDonnell JM and Korsmeyer SJ: BCL-2 family members and the mitochondria in apoptosis. Genes Dev 13: 1899-1911, 1999.

35. Coultas L and Strasser A: The role of the Bcl-2 protein family in cancer. Semin Cancer Biol 13: 115-123, 2003.

36. Dejean LM, Martinez-Caballero S, Guo L, et al: Oligomeric Bax is a component of the putative cytochrome $\mathrm{c}$ release channel MAC, mitochondrial apoptosis-induced channel. Mol Biol Cell 16: 2424-2432, 2005.

37. Youle RJ and Strasser A: The BCL-2 protein family: opposing activities that mediate cell death. Nat Rev Mol Cell Biol 9: 47-59, 2008.

38. Ren D, Yang H and Zhang S: Cell death mediated by MAPK is associated with hydrogen peroxide production in Arabidopsis. J Biol Chem 277: 559-565, 2002.

39. Cho SJ, Rhee DK and Pyo S: Allicin, a major component of garlic, inhibits apoptosis of macrophage in a depleted nutritional state. Nutrition 22: 1177-1184, 2006.

40. Ji C, Ren F and Xu M: Caspase- 8 and $\mathrm{p} 38^{\mathrm{MAPK}}$ in DATS-induced apoptosis of human CNE2 cells. Braz J Med Biol Res 43: 821-827, 2010.

41. Inoue M, Sakaguchi N, Isuzugawa K, Tani H and Ogihara Y: Role of reactive oxygen species in gallic acid-induced apoptosis. Biol Pharm Bull 23: 1153-1157, 2000.

42. Kannan K and Jain SK: Oxidative stress and apoptosis. Pathophysiology 7: 153-163, 2000.

43. Circu ML and Aw TY: Reactive oxygen species, cellular redox systems, and apoptosis. Free Radic Biol Med 48: 749-762, 2010.

44. Cohen GM: Caspases: the executioners of apoptosis. Biochem J 326: 1-16, 1997. 